\title{
Correction to: Stereotactic Radiofrequency Ablation of Breast Cancer Liver Metastases: Short- and Long-Term Results with Predicting Factors for Survival
}

\author{
Peter Schullian $^{1} \cdot$ Edward Johnston $^{2}$ - Gregor Laimer ${ }^{1}$ - Daniel Putzer ${ }^{1}$ • \\ Gernot Eberle $^{1} \cdot$ Yannick Scharll $^{1} \cdot$ Claudia Ianetti-Hackl $^{3} \cdot$ Reto Bale $^{1}$ (D)
}

Published online: 21 June 2021

(C) Springer Science+Business Media, LLC, part of Springer Nature and the Cardiovascular and Interventional Radiological Society of Europe (CIRSE) 2021

\section{Correction to: Cardiovasc Intervent Radiol https://doi.org/10.1007/s00270-021-02820-6}

The name of the sixth author is listed incorrectly in the published article as Yannick Scharrl.
The correct name is Yannick Scharll.The correct name is Yannick Scharll.

The original article has been corrected.

Publisher's Note Springer Nature remains neutral with regard to jurisdictional claims in published maps and institutional affiliations.

The original article can be found online at https://doi.org/10.1007/ s00270-021-02820-6.

Reto Bale

reto.bale@i-med.ac.at

1 Department of Radiology, Section of Interventional Oncology - Microinvasive Therapy (SIP), Medical University of Innsbruck, Anichstr. 35, Innsbruck 6020, Austria

2 Royal Marsden Hospital, 203 Fulham Road, Chelsea, London SW3 6JJ, UK

3 Transplant and Thoracic Surgery, Center of Operative Medicine, Medical University of Innsbruck, Anichstr. 35, Innsbruck 6020, Austria 